\title{
An examination of response bias in multiletter matching
}

\author{
ROBERT W. PROCTOR, K. VENKATA RAO, and PHILIP W. HURST \\ Auburn University, Auburn, Alabama
}

\begin{abstract}
When pairs of letters or letter strings are classified as "same" or "different," correct "same" responses often are faster than correct "different" responses. Researchers have disagreed about the importance of this fast-same phenomenon, with some regarding it as indicative of a critical, basic characteristic of pattern recognition processes and others regarding it as a byproduct of response criteria. The present experiments examined this issue for multiletter matching. Two procedures were used, one in which response bias was manipulated by varying the proportion of same pairs and another in which string length effects were examined for a situation designed to minimize premature termination of the comparison process. The procedures provided converging results, indicating that, although the fast-same effect customarily obtained with simultaneous presentation of the pair of letter strings is likely due to response bias, the effect obtained with successive presentation is not. With successive presentation, same strings have a processing advantage over different strings that apparently is due, at least in part, to facilitation in the encoding of the second string.
\end{abstract}

In pattern-matching tasks, subjects classify pairs of stimuli as either "same" or "different." When the stimuli within the pairs are single letters, multiletter strings, or multidimensional nonalphanumeric stimuli, correct "same" responses often are faster than correct "different" responses (Krueger, 1978; Nickerson, 1978). The fast-same phenomenon has been the subject of intensive investigation because it is inconsistent with most feature-analytic models of the comparison process. These models predict that, if anything, "different" responses should be faster than "same" responses, because a single difference in component features is sufficient to indicate "different," whereas all features must match to indicate "same" (Bamber, 1969; Nickerson, 1965). The violation of this prediction of feature-analytic models suggests that the fast-same phenomenon may provide a fundamental insight into the processes that underlie matching-task performance.

Because of its apparent importance, the fast-same phenomenon has been the focus of several patternmatching models (Bamber, 1969; C. W. Eriksen, O'Hara, \& B. A. Eriksen, 1982; Krueger, 1978; Proctor, 1981; Taylor, 1976). One exception is the model developed by Ratcliff (1981), which dealt only cursorily with the fast-same phenomenon, because

\footnotetext{
This research was supported in part by a research Grant-in-Aid from Auburn University. We would like to thank Dennis Hanks, Stephanie Ford, Mitch Jones, Jim Parker, and Hunter Peak for assistance in the collection and scoring of the data. We would also like to express our gratitude to T. Gilmour Reeve and Peter Harzem for allowing us use of their computer facilities and to James McAlarney III for programming the experiments. Requests for reprints should be sent to Robert W. Proctor, Department of Psychology, Auburn University, Auburn, AL 36849.
}

the phenomenon was regarded as "little more than a criterion effect"' (p. 570). Ratcliff and Hacker (1981) more strongly stated this position in a study devoted entirely to demonstrating that the relationship between "same" and "different" response latencies is affected by response criteria. In their most critical experiment, subjects compared pairs of successively presented four-letter strings that either were identical (same) or different at one to four positions (different). Response bias was manipulated by instructing subjects in different sessions to respond "same" only when sure or to respond "different" only when sure. Whereas "same" responses were faster than "different" responses when subjects were biased against responding "different," the relationship was reversed when subjects were biased against responding "same." Ratcliff and Hacker interpreted the effects of their bias instructions in terms of the relative settings for "same" and "different" response criteria in a sequential sampling process. That is, the criterion for a "same" response was assumed to be set relatively higher than that for a "different" response when subjects were biased against responding "same," with the relative settings of the criteria reversed when the subjects were biased against responding "different."

Although the usual fast-same effect was eliminated, and a fast-different effect obtained, when subjects were biased against responding "same," the advantage for "different" responses in this situation was $\mathbf{5 2} \mathrm{msec}$ less than the advantage for "same" responses that obtained when subjects were biased against responding "different." Ratcliff and Hacker (1981) concluded that this overall reaction-time advantage for "same" responses was attributable only 
to a residual bias in the settings of the "same"-"different" response criteria. They stated, "Thus, from our point of view, this 52 -msec difference is simply a function of the relative bias attained by our particular subjects with these particular stimuli and nothing more" (Ratcliff \& Hacker, 1982, p. 603, italics added).

Prior to Ratcliff and Hacker's (1981) study, several other investigators considered the possibility that the fast-same phenomenon might reflect "nothing more" than response bias (e.g., Grill, 1971; Taylor, 1976). The consensus has been, however, that response bias accounts are inadequate (see Krueger, 1977, and Nickerson, 1972; for reviews that reached this conclusion). All the evidence will not be recounted here, but one of the most important findings is that in certain situations the relative proportions of incorrect "same" and "different" responses do not conform to the expectations of response bias accounts. Response bias accounts of the fast-same phenomenon that argue either that subjects have a relatively lower "same" criterion in a continuous sampling process (e.g., Ratcliff \& Hacker, 1981) or that subjects prematurely terminate an analytic comparison prior to locating a mismatching feature (e.g., Grill, 1971; Taylor, 1976) predict that false-"same" responses (incorrectly responding "same" to different pairs) should outnumber false-"different" responses (incorrectly responding "different" to same pairs). To the contrary, for single-element stimuli (such as single-letter pairs), false-"different" responses tend to predominate (Krueger, 1977, 1978).

Although the evidence indicates that the fast-same phenomenon cannot be attributed entirely to response bias, Ratcliff and Hacker's (1981) demonstration, as well as similar ones by Coltheart and Curthoys (1968), Downing (1971), and Krueger and Shapiro (1981a), clearly shows that response criteria do influence the relationship between "same" and "different" response latencies. This demonstration indicates that care must be taken to evaluate the role that response criteria play for all situations in which the fast-same phenomenon has been obtained, with a particular emphasis toward discerning whether there are situations in which the effect is attributable entirely to response bias. If there are such situations, distinguishing them from those situations in which it is not should help clarify the processes, other than response bias, that contribute to the phenomenon.

In addition to providing a means for examining the specific effects of criterion shifts, the bias methodology used by Ratcliff and Hacker (1981) allows evaluation of the extent to which a fast-same effect that is independent of bias exists. Proctor and Rao (1982) indicated that when the relative bias to respond "same" or "different" is manipulated symmetrically (as in Ratcliff \& Hacker's, 1981, Experiment 1), a processing advantage for same pairs should occur as a main effect in response latencies. That is, overall, correct "same" responses should be faster than correct "different" responses. As indicated previously, such a difference was apparent in Ratcliff and Hacker's experiment, but, because false"same" errors outnumbered false-"different" errors, a response bias interpretation is still viable (Ratcliff \& Hacker, 1982).

Proctor and Rao (1983) therefore used a variation of the bias methodology to obtain results that were inconsistent with the viewpoint that the fast-same phenomenon is entirely attributable to bias in the settings of "same" and "different" response criteria. Single-letter pairs, rather than multiletter pairs, were used as stimuli because they are relatively simple stimuli that have been used extensively to examine the fast-same effect. Response bias was manipulated by varying the proportion of same and different pairs included in blocks of trials. Four different blocks of 100 trials that contained $80,60,40$, or 20 same pairs were employed. As in previous studies, the relative latencies of "same" and "different" responses varied across proportion blocks in the manner expected from the bias manipulation. Most importantly, there was an overall reaction-time advantage for correct "same" responses that could not be attributed simply to response bias. That is, the fast-same effect was paired with an overall preponderance of false"different" errors. Although the fast-same phenomenon was obtained with both simultaneous and successive presentation of the letters, the effect was greater in the latter case $(M=26.4 \mathrm{msec})$ than in the former case $(M=9.8 \mathrm{msec})$, as is often found in reaction-time studies.

The experiments reported in the present article represent a continuation of the investigation begun by Proctor and Rao (1983). Having obtained evidence that response bias, alone, cannot account for the fast-same phenomenon obtained with singleletter pairs, the next step was to determine whether such is also the case for multiletter pairs. The potential role for response bias is much greater in multiletter matching than in single-letter matching, because false-"same" errors predominate (e.g., Proctor \& Hurst, 1982; Silverman, 1973; Taylor, 1976). The relatively large number of errors of this type that occur for strings that differ at only one position has been interpreted as indicating that the search process is often prematurely terminated, and a "same" response made, before all comparisons are completed (Taylor, 1976). Thus, response bias is apparently a factor contributing to the fast-same phenomenon for multiletter comparisons, although authors have argued that it cannot account for the entire effect (Bamber \& Paine, 1973; Taylor, 1976).

Two different methods were used in the present study to address the role of response bias in multiletter matching. The first method involved manipulat- 
ing bias in the same manner that Proctor and Rao (1983) did for single-letter pairs. The second method involved examining string length effects in a situation designed to minimize errors, and, thus, premature terminations of the comparison process. For each approach, conditions were examined in which the two strings were presented successively and in which they were presented simultaneously. The two methods of presentation were examined (1) because with single-letter pairs the fast-same phenomenon has been obtained most consistently and reliably with successive presentation, and (2) because a substantial portion of the fast-same effect obtained with successive presentation is due apparently to a priming or facilitation factor that is not involved with simultaneous presentation (Krueger, 1983; Krueger \& Shapiro, 1981b; Proctor, 1981; Proctor \& Rao, 1983).

\section{EXPERIMENT 1: MANIPULATING RESPONSE BIAS}

Response bias was manipulated in Experiment 1 by varying whether blocks of 100 trials contained 80,60 , 40 , or 20 same pairs, with the remaining pairs being different. Only four-letter strings were used as stimuli, with same pairs being identical at all positions and different pairs differing at only one position. Different pairs were restricted to this type because these one-different pairs provide the most appropriate baseline against which to evaluate responses to same pairs (Proctor \& Hurst, 1982).

Experiment 1 was, thus, similar to the experiment conducted by Ratcliff and Hacker (1981); only fourletter strings were used and bias was manipulated between blocks of trials. The experiment differed from that of Ratcliff and Hacker in that bias was manipulated by varying the proportion of same and different pairs, rather than by instructions alone, and in that strings that differed at only one position were used as different pairs, rather than strings that differed at one, two, three, or four positions. For some subjects, the two strings were presented successively, as in Ratcliff and Hacker's experiment, whereas for others they were presented simultaneously.

\section{Method}

Subjects. Sixteen subjects were tested with successive presentations of the two letter strings, and 16 different subjects were tested with simultaneous presentation. Although the latter group of subjects was tested after the former group, the data are described together for ease of presentation. Each subject was tested in two sessions on successive days.

Apparatus. The experiment was conducted on a Radio Shack TRS-80 Model III microcomputer. Stimuli were members of the standard character set presented on the display screen. Exposure durations, interstimulus intervals, and intertrial intervals were controlled by the computer.

Responses were made by pressing one of two response keys on the computer's keyboard (a standard typewriter keyboard). Half of the subjects responded "same" by pressing the Z key with the left index finger and "different" by pressing the ? key with the right index finger. For the other half of the subjects, this pairing was reversed. Reaction times were recorded to the nearest millisecond by the computer.

Stimuli. Stimuli were pairs of four-letter strings. The strings were composed from uppercase versions of all letters of the alphabet, excluding the vowels $A, E, I, O$, and $U$. Within a given string, no letters were repeated. Pairs of strings were either identical (the same letters in the same positions) or differed at only one position. For these latter pairs, the difference occurred equally often at all four positions, with the second string generated by replacing the letter in the critical position of the first string with a new letter that was different from any other letter in the string.

The subjects viewed the display screen at a distance of approximately $50 \mathrm{~cm}$. Individual letters subtended a visual angle of approximately $.57 \mathrm{deg}$ in height and $.34 \mathrm{deg}$ in width. Letter strings were displayed horizontally, with spaces of approximately $.34 \mathrm{deg}$ between each letter, thus subtending an angle of approximately 2.38 deg. The pair of strings on a trial were presented centered about a fixation spot (an asterisk). One string appeared approximately $.68 \mathrm{deg}$ above the center of the spot, whereas the other string appeared approximately $.68 \mathrm{deg}$ below the center of the spot.

Within each session, a block of 20 practice trials was presented (10 same pairs and 10 different pairs), followed by four blocks of 100 trials. The proportion of same and different pairs was varied between blocks, such that there were either 80 same and 20 different pairs, 60 same and 40 different pairs, 40 same and 60 different pairs, or 20 same and 80 different pairs. These conditions are hereafter referred to as the $80 / 20,60 / 40,40 / 60$, and $20 / 80$ conditions, respectively. Each block of trials was preceded by two warm-up trials involving the predominant type of pair in the two extreme proportion blocks and one pair of each type in the two intermediate blocks. The order in which the blocks were presented was counterbalanced across subjects, with each subject receiving the same order in both sessions.

Procedure. The fixation spot remained on at all times other than when it offset for $250 \mathrm{msec}$ as a warning for the upcoming trial. Two hundred and fifty milliseconds after the spot reappeared, the stimuli were presented. For the successive presentation condition, the string above fixation occurred for $500 \mathrm{msec}$, followed by a blank interval of $500 \mathrm{msec}$, and then the string below fixation; for the simultaneous presentation condition, both strings occurred simultaneously. The subject's response terminated the second of the two successively presented strings or both of the simultaneously presented strings. One second elapsed between the response and the flicker of the fixation spot that signaled the start of the next trial.

Prior to the first session, the subjects were given general instructions regarding the matching task, indicating that they were to respond "same" if the letter strings matched at all positions and "different" if the strings differed at one position. The instructions stressed responding rapidly at the expense of making some errors. Furthermore, the subjects were also told that the proportion of same and different pairs would vary between blocks of trials and that they should take advantage of this knowledge. Prior to each block, the proportion of same and different pairs was shown on the display screen. This information remained on the screen until the subject initiated the block of trials.

\section{Results}

For the primary analyses, the position of the mismatching letter in different pairs was disregarded. Position effects for these pairs are examined following presentation of all three experiments. The proportions of errors and mean correct reaction times were obtained for each subject as a function of type of pair (same, different), proportion block 
$(80 / 20,60 / 40,40 / 60,20 / 80)$, method of presentation (simultaneous, successive), and session $(1,2)$. Responses faster than $200 \mathrm{msec}$ or slower than $2 \mathrm{sec}$ $(<1 \%)$ were excluded from the computation of mean reaction times. Because similar patterns were evident in both sessions, the data were pooled across sessions. The means of these data are shown in Figure 1.

Responses were slower with simultaneous presentation $(M=1,107 \mathrm{msec})$ than with successive presentation $(\mathrm{M}=667 \mathrm{msec})[\mathrm{F}(1,15)=108.5, \mathrm{p}<.001]$, but also were more accurate (proportions of errors equal .079 and .147 , respectively) $[\mathrm{F}(1,15)=10.3$, $\mathrm{p}<.01$ ]. More importantly, as indicated by significant interactions of proportion block $\times$ pair type for both reaction times $[F(3,45)=7.84, p<.001]$ and errors $[F(3,45)=15.6, p<.001]$, the proportion manipulation was effective at altering response bias and, moreover, was equally effective for simultaneous and for successive presentation (proportion block $\times$ pair type $\times$ method of presentation, Fs $<$ 1.0). For both methods of presentation, the proportion of false-"same" responses increased and the proportion of false-"different" responses decreased as the percentage of same pairs increased. Reaction times for correct "same" responses also decreased relative to those for correct "different" responses as the percentage of same pairs increased.

Overall, however, more errors were made to different pairs $(M=.143)$ than to same pairs $(M=.083)$ $[F(1,15)=36.0, p<.001]$, and there was a tendency for correct responses to be faster to different pairs than to same pairs $(\mathrm{M}=882 \mathrm{msec}$ and $\mathrm{M}=893 \mathrm{msec}$, respectively) $[F(1,15)=4.49, p<.10]$. Although the error difference held for both simultaneous and successive presentation (pair type $\times$ method, $F<1.0$ ), the reaction time difference did not [pair type $x$ method, $F(1,15)=10.9, p<.01]$. With simultaneous presentation, responses were faster to different pairs $(\mathrm{M}=1,067 \mathrm{msec})$ than to same pairs $(\mathrm{M}=1,147 \mathrm{msec})$ $[F(1,15)=14.6, p<.005]$. With successive presentation, the relationship was reversed: Responses were faster to same pairs $(M=638 \mathrm{msec})$ than to different pairs $(M=695 \mathrm{msec})[\mathrm{F}(1,15)=18.5, \mathrm{p}<.001]$.

The predominance of errors to different pairs (false-"same" responses) that occurred with both simultaneous and successive presentation is consistent with an overall bias to respond "same." Thus, the reaction time advantage for different pairs obtained with simultaneous presentation can unambiguously be attributed to a factor other than bias, whereas the reaction time advantage for same pairs that occurs with successive presentation cannot be.

Finally, the main effect of proportion block was significant for the reaction time data $[F(3,45)=11.2$, $\mathrm{p}<.001]$ but not for error rate $(\mathrm{F}<1.0)$. The significant effect on reaction time reflects an overall increase in latency as same pairs became less frequent (means equal 840, 883, 916, and $909 \mathrm{msec}$ for the $80 / 20,60 / 40,40 / 60$, and $20 / 80$ conditions, respectively). This main effect is attributable to the fact that "same" reaction times were influenced much more by the proportion manipulation than were "different" reaction times. The implication of this result is examined in General Discussion.
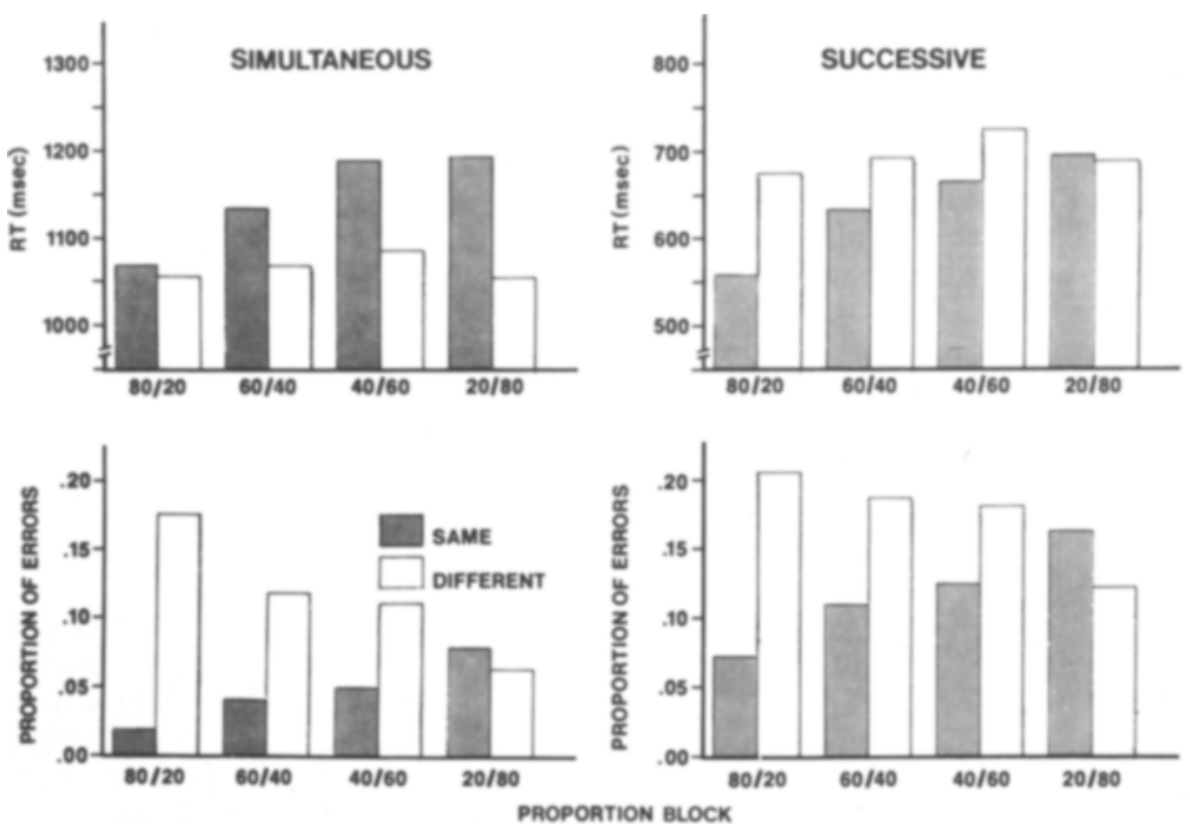

Figure 1. Mean reaction time (RT) and proportion of errors in Experiment 1 as a function of method of presentation, proportion block, and type of pair. 


\section{Discussion}

With both simultaneous and successive presentation, varying the proportion of same and different pairs contained in blocks of trials altered the relative bias to respond "same" or "different." As the proportion of same pairs increased, the relative number of false-"same" errors increased and the relative latency of correct "same" responses decreased.

Over and above the effects of the bias manipulation, there were main effects for both error rate and reaction time. For both methods of presentation, false-"same" errors outnumbered false-"different" errors, indicating a bias to respond "same." With simultaneous presentation, this predominance of false-"same" errors was somewhat surprisingly paired with faster responses to different pairs than to same pairs, indicating an advantage for the processing of different pairs that cannot be attributed to response bias. With successive presentation, however, responses were faster to same pairs than to different pairs. This latency advantage for correct "same" responses, along with the predominance of false"same" errors, replicates the findings of Ratcliff and Hacker (1981) and cannot be interpreted unambiguously as indicating an advantage in the processing of same pairs other than one of response bias.

One outcome, however, suggests that the latency advantage for same pairs with successive presentation is not attributable solely to response bias. Krueger and Shapiro (1981b) proposed that the larger fast-same effect obtained with successive presentation than with simultaneous presentation for singleletter pairs is due to a bias shift. They reasoned that this increased advantage for same pairs, thus, should be accompanied by a relative increase in false"same" errors. Tests of this prediction with singleletter pairs have proved negative (Chignell \& Krueger, 1984; Krueger, 1983; Proctor \& Rao, 1983), indicating that the larger difference obtained with successive presentation is not due to a change in bias.

In the present experiment, there was also no increased tendency to make false-"same" errors rather than false-"different" errors when the two strings were presented successively, as opposed to simultaneously. This suggests that the latency advantage obtained for same pairs with successive presentation, but not with simultaneous presentation, is attributable to a factor other than response bias. Because of the large differences between the two methods of presentation in the overall levels of reaction time and accuracy, however, this evidence must be viewed with caution. Experiment 2 was conducted to provide additional evidence that the fast-same phenomenon obtained with successive presentation is not due solely to response bias.

\section{EXPERIMENT 2: \\ MANIPULATING RESPONSE BIAS FOR SUCCESSIVELY PRESENTED THREE-LETTER STRINGS}

The pattern of responding obtained with successive presentation in Experiment 1, faster responses to same pairs than to different pairs and a predominance of false-"same" errors, is consistent with a response-bias account of the fast-same phenomenon. That is, whenever false-"same" errors predominate, it is impossible to exclude response bias as an account of the latency advantage. Our concern was, thus, in obtaining data from a situation that would not produce the predominance of false-"same" errors.

Examination of the literature indicates that, for most studies in which string length is varied, a large upsurge in the number of false-"same" responses occurs for four-letter strings (see, e.g., Experiment 3 of the present article). Therefore, with the bias methodology, three-letter strings likely would not show a predominance of false-"same" errors. Experiment 2 was, thus, a replication of the successive presentation condition from Experiment 1, using three-letter instead of four-letter strings.

\section{Method}

Sixteen new subjects from the same subject pool as in Experiment 1 participated in the experiment. The primary change in method was the use of three-letter strings rather than four-letter strings. To have the mismatching pair occur equally often at all positions, the number of pairs in each block was increased to 105 , with the proportions of same and different pairs kept the same as in the previous experiment. For ease of reference, we shall still refer to these conditions as $80 / 20,60 / 40,40 / 60$, and $20 / 80$.

\section{Results}

The mean proportions of errors and mean reaction times are shown in Figure 2. As anticipated, both measures showed an effect of the bias manipulation, with the proportion of errors and correct reaction time increasing for same pairs relative to different pairs as the proportion of same pairs decreased $[F(3,45)=16.4, p<.001$, for errors, and $F(3,45)=$ $17.9, \mathrm{p}<.001$, for reaction time]. As in Experiment 1 , the bias manipulation had a greater effect on reaction time to same pairs than on that to different pairs, resulting in a main effect for proportion block $[F(3,45)=8.36, p<.001]$.

Most importantly, the error-rate data showed no overall difference between the proportions of errors made to same pairs $(M=.058)$ and to different pairs $(\mathrm{M}=.060)(\mathrm{F}<1.0)$. Despite this lack of a difference in error rate, responses to same pairs $(M=632 \mathrm{msec})$ were reliably faster overall than responses to different pairs $(\mathrm{M}=691 \mathrm{msec})[\mathrm{F}(1,15)=20.5, \mathrm{p}<.001]$. 

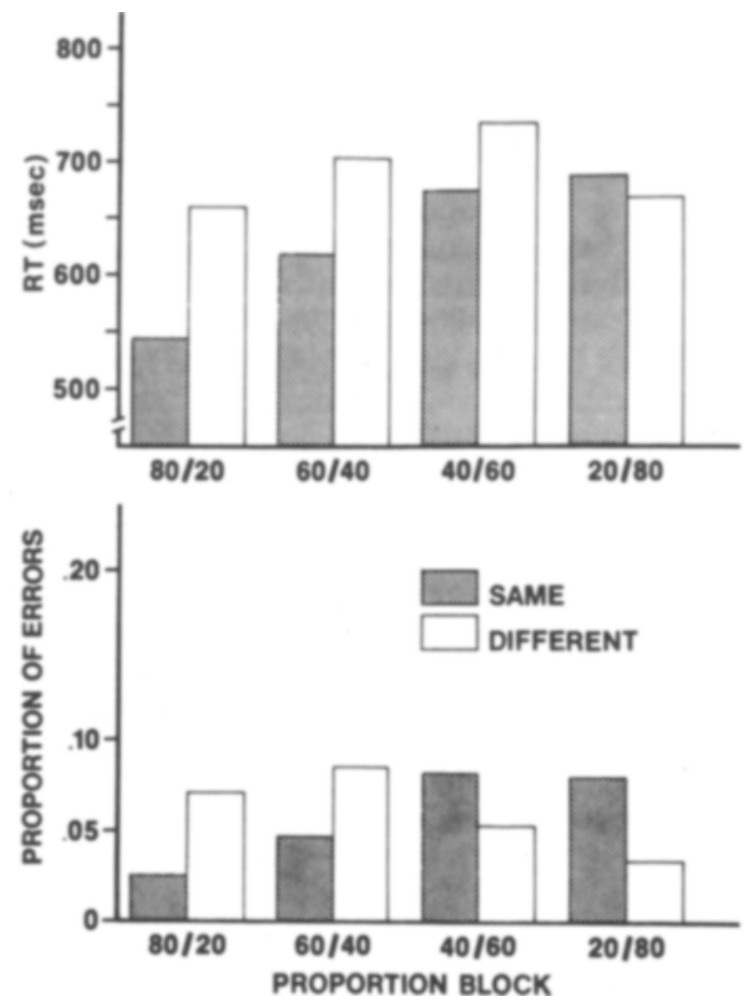

Figure 2. Mean reaction time (RT) and proportion of errors in Experiment 2 as a function of proportion block and type of pair.

Thus, the experiment clearly shows a fast-same effect when the error-rate data show no apparent overall bias to respond "same."

\section{EXPERIMENT 3: MINIMIZING ERRORS}

Much interest in multiletter matching has focused on the finding that as string length increases from one to four items, reaction time to same pairs usually increases at approximately the same or at a slightly lesser rate than does reaction time to different pairs that differ at only one position. Because "same" responses are faster than "different" responses for single-letter pairs, the reaction-time functions are approximately parallel, with the function for same pairs lying below that for different pairs (Bamber, 1969; Proctor, 1981; Proctor \& Hurst, 1982; Taylor, 1976).

The results obtained in Experiments 1 and 2 with successively presented four-letter strings are consistent with this finding. That is, responses were faster overall to same pairs than to different pairs. The results obtained with simultaneous presentation, however, are not consistent with the finding of approximately parallel functions. Responses were slower overall to same pairs than to different pairs.

The reason for this discrepancy between Experiment 1 , which found faster responses to different pairs with simultaneous presentation, and previous experiments, which found faster responses to same pairs with simultaneous presentation (Eichelman, 1970; Henderson \& Henderson, 1975; Proctor \& Hurst, 1982; Silverman, 1973; Silverman \& Goldberg, 1975), most likely lies in the relatively high proportion of false-"same" errors made in previous studies." The proportion of false-"same" errors increases greatly with string length, whereas the proportion of false"different" errors remains relatively constant. This progressively greater tendency to incorrectly respond "same" at longer string lengths, which is particularly apparent for strings that differ at only one position, has been attributed, as indicated previously, to termination of the comparison process on some trials prior to the subject's either locating a mismatching letter pair or completing all comparisons (Taylor, 1976).

Consideration of the prototypical procedure used in studies of multiletter matching indicates that it, in fact, emphasizes this premature termination (see also, Grill, 1971). Generally, pairs of the various lengths (usually one to four letters) are randomly intermixed in the presentation sequence. In addition, for string lengths greater than one, different pairs are often divided equally among ones that differ at one position, two positions, etc., up to the maximum allowed by the string length (i.e., all letters). Thus, pairs that differ minimally (at only one position) are relatively rare, particularly at longer string lengths, and this rarity biases subjects to classify them as "same."

Experiment 3 was conducted to reconcile the results of Experiment 1 with the data on the effect of string length for simultaneously presented letter strings by manipulating string length in an experiment designed to minimize errors and, thus, premature terminations. To diminish the premature termination problems of previous studies, trials were blocked by string length, and all different pairs differed at only one position. Thus, subjects knew the string length for the pairs within a block of trials, and they also knew that the different pairs would be of the most difficult type. Furthermore, the subjects were told to try to make no errors, again with the intent of minimizing the tendency to initiate "same" responses prior to the completion of processing. This general procedure was also replicated with successive presentation of the pairs to determine whether the differences between successive and simultaneous presentation apparent in Experiment 1 were maintained with the high-accuracy procedure.

\section{Method}

Subjects. Twenty-four students who had not participated in Experiments 1 and 2 served as subjects. Half of these subjects were tested with simultaneous presentation, and, subsequently, the other half were tested with successive presentation.

Apparatus and Stimull. The experiments were conducted on a Radio Shack TRS-80 Model I microcomputer, rather than the Model III used in Experiment 1. The display of the Model I is sim- 
ilar to that of the Model III. All timing was controlled by the computer, and the assignment of responses to keys on the keyboard was the same as in the previous experiments.

Stimuli were pairs of uppercase letter strings composed from the set $B, D, F, G, H, L, M, N, R$, and $T$. The strings were $1,2,3$, or 4 letters in length, with no letters repeated within a string, and both strings in a pair were always of the same length. The two strings in a pair were either the same at all positions (same pairs) or different at only one position (different pairs). For different pairs, the mismatching letter pair occurred equally often at all positions. These pairs were generated by randomly replacing the letter in the appropriate position of one string with a letter not contained in that string.

As in Experiments 1 and 2, the letter strings were displayed horizontally, with one string above and the other below a small fixation spot. The individual letter sizes were similar to those in Experiments 1 and 2 (approximately $.34 \mathrm{deg}$ in width), with a gap of approximately .1 deg between each letter in the multiletter strings. Thus, the visual angle subtended by the 2-, 3-, and 4-letter strings was approximately .78, 1.22, and $1.66 \mathrm{deg}$, respectively. As in Experiments 1 and 2, the bottom of the upper string and the top of the lower string were approximately $.68 \mathrm{deg}$ above and below the center of the fixation spot, respectively.

Within each session, the pairs of letter strings were presented in four blocks in which string length was held constant. For string lengths of 1, 2, and 4, the blocks contained 32 same pairs and 32 different pairs. In order to have the mismatching letter pair for different pairs occur equally often at each position, an additional same and different pair were included for the string length of 3 , thus making 33 same and 33 different pairs for this block. Each block began with two additional warm-up trials that were not included in the data analyzed.

Procedure. The experiment consisted of three sessions, the first of which was counted as practice. Each subject received the blocks in different orders in each session, with the three orders selected from the four possible in a Latin square. Order was counterbalanced across subjects for each session.

The trial sequence was similar to that of Experiments 1 and 2. Each trial began with a 500 -msec warning interval indicated by a flicker of the fixation spot. Following this interval, the pair of letter strings was presented. For half of the subjects, the two strings were presented simultaneously, whereas for the remaining subjects the top string was presented for $500 \mathrm{msec}$, then followed, after a 500 -msec blank interval, by the bottom string. Both of the simultaneously presented strings and the second of the successively presented strings were terminated by the subject's response. An intertrial interval of $3 \mathrm{sec}$ separated successive trials.

The subjects were given general instructions regarding the matching task, indicating that they were to respond "same" if the letter strings matched at all positions and "different" if the strings differed at one position. The instructions stressed accuracy in responding. The subjects were told that they should try to make essentially no errors, but to respond as rapidly as possible within that constraint.

The subjects were also told that string length would be held constant within a block of trials. Prior to each block, the string length was indicated on the display screen. This information remained on the screen until the subject initiated the block of trials.

\section{Results}

One subject in each of the method-of-presentation conditions produced error rates approximately twice those of any other subject. Because our intent was to examine reaction time when errors were minimal, these two subjects were excluded from the data analyses. However, their inclusion would not have altered the major findings regarding the reaction time measure. The data were collapsed across the two test sessions because similar patterns were apparent in each.
Error rate. The mean proportion of errors as a function of type of pair, string length, and method of presentation is shown in Table 1. Although accuracy had been stressed in the instructions, the subjects did not perform with perfect accuracy. However, the overall proportion of errors $(\mathrm{M}=.027)$ was less than is often obtained in studies of multiletter matching.

An analysis of variance indicated significant main effects for string length $[F(3,30)=11.8, p<.001]$ and type of pair $[F(1,10)=12.77, p<.01]$, as well as a significant interaction between these two variables $[F(3,30)=9.68, p<.001]$. Basically, these effects reflect an increase, limited to different pairs, in the proportion of errors as string length increased. This increase in false-"same"' errors occurred primarily for four-letter strings and was substantially less than is obtained customarily. Method of presentation showed neither a main effect nor interactions with the other two variables, indicating that subjects maintained accuracy equally well for both methods of presentation.

To summarize, the experimental procedures were of intermediate effectiveness at eliminating false"same" errors. Although performance did not approach perfect accuracy, the total proportion of errors and the proportion of false-"same" errors were less than usually obtained, particularly for different pairs at the longer string lengths. As will be seen, this relatively low error rate resulted in a pattern of reaction times for simultaneous presentation that is different from those obtained in previous studies.

Reaction time. The mean reaction times for correct responses are shown in Figures 3 and 4. A threefactor analysis of variance (pair type $X$ string length $x$ method of presentation) showed significant effects (ps $<.025$ ) for all main effects and interactions except the main effect for pair type $(F<1.0)$. The significant main effects for method of presentation and string length indicate that, as usually obtained, responses were slower with simultaneous presentation than with successive presentation and that response latency increased with increases in string length. The remaining results are best conveyed by separate analyses for each method of presentation.

Table 1

Proportion of Errors in Experiment 3 as a Function of String Length, Type of Pair, and Method of Presentation

\begin{tabular}{lllll}
\hline & \multicolumn{4}{c}{ String Length } \\
\cline { 2 - 4 } Type of Pair & 1 & 2 & 3 & 4 \\
\hline \multirow{4}{*}{$\begin{array}{l}\text { Simultaneous Presentation } \\
\text { Same }\end{array}$} & .023 & .011 & .019 & .018 \\
Different & .014 & .023 & .036 & .058 \\
& Successive Presentation & \\
Same & .027 & .026 & .025 & .024 \\
Different & .020 & .020 & .026 & .068 \\
\hline
\end{tabular}




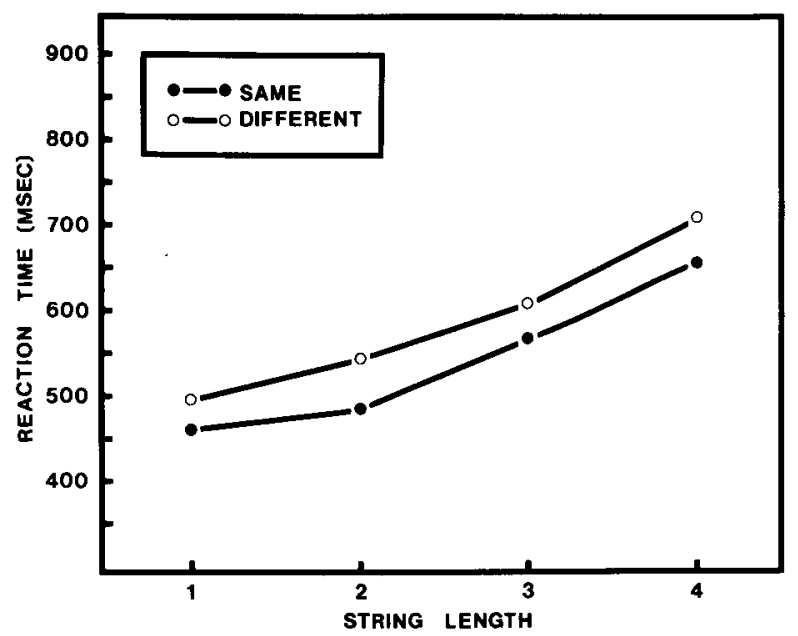

Figure 3. Mean reaction time as a function of string length and type of pair for successively presented pairs in Experiment 3.

As Figure 3 shows, the pattern of results for successive presentation was similar to that of previous studies in which string length was manipulated. Reaction time increased with increases in string length $[F(3,30)=14.0, p<.001]$, and responses were faster to same pairs than to different pairs $[F(1,10)=14.6$, $\mathrm{p}<.005]$. Most importantly, there was no interaction of pair type with string length $(F<1.0)$. Thus, the reaction time advantage for same pairs was evident to an equivalent extent at all string lengths.

As Figure 4 shows, the pattern of results for simultaneous presentation was quite different. Although reaction time increased with increases in string length $[F(3,30)=55.2, p<.001]$, as it also did for successive presentation, responses were slower to same pairs than to different pairs $[\mathrm{F}(1,10)=9.32, \mathrm{p}<.025]$, and type of pair interacted significantly with string length $[F(3,30)=14.97, p<.001]$. In contrast to the results obtained with successive presentation, and with simul-

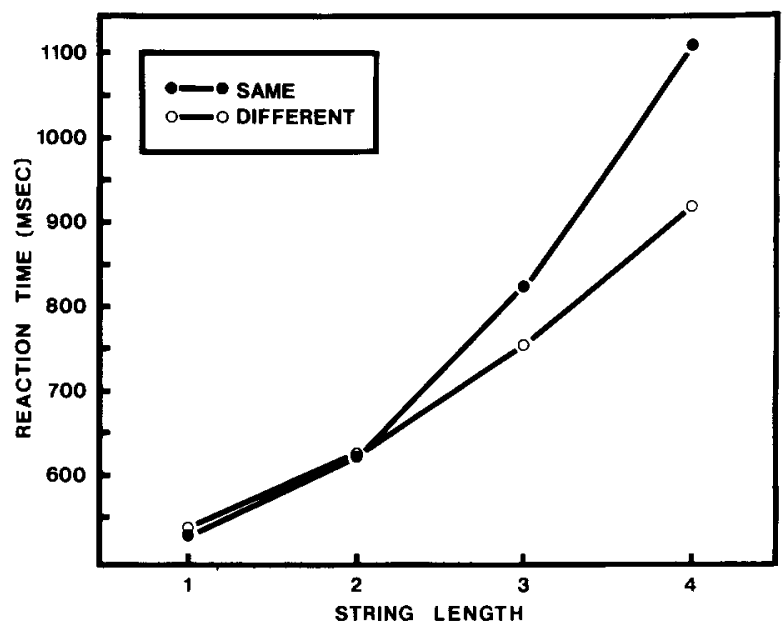

Figure 4. Mean reaction time as a function of string length and type of pair for simultaneously presented pairs in Experiment 3. taneous presentation in previous studies, the effect of string length was much greater on the response latency to same pairs than on the latency to different pairs.

Trend analyses indicated that for each method of presentation, the bulk of the variance attributable to string length was located in the linear component. With successive presentation, only the linear component was significant for both "same" $[F(1,30)$ $=37.2, p<.001]$ and "different" $[F(1,30)=34.4$, $\mathrm{p}<.001]$ response latencies. With simultaneous presentation, only the linear component was significant for "different" reaction times $[F(1,30)=158.8$, $\mathrm{p}<.001$ ], whereas for "same" reaction times there was a reliable quadratic component $[F(1,30)=6.76$, $\mathrm{p}<.025]$ in addition to the linear component $[F(1,30)$ $=135.2, \mathrm{p}<.001]$. This quadratic component reflects a positive acceleration to the function that is present, to a lesser extent, for the other functions, as well.

The lack of interaction between string length and pair type for successive presentation indicates that the best fitting straight lines for the same and different functions have approximately equivalent slopes. For simultaneous presentation, the slope of the function for different pairs was .655 that of the slope of the function for same pairs (the mean ratio for the individual subjects' functions was .688). This slope relationship for simultaneous presentation is generally consistent with the view that "different" responses can be executed whenever a single mismatching letter pair is detected. In fact, the slope ratio is relatively close to the ratio of .5 that is predicted by the standard serial self-terminating model (Bamber, 1969) and its parallel equivalent (Taylor, 1976).

\section{Discussion}

The results of Experiment 3 are in accord with those of Experiments 1 and 2. With bias to respond "same" minimized, correct "different" responses are faster than correct "same" responses, when the letter strings are presented simultaneously. However, when presented successively, correct "same" responses are faster than correct "different" responses. Thus, as in Experiments 1 and 2, the fastsame phenomenon was apparent for successively presented letter strings, but not for simultaneously presented strings.

As in Experiment 1, the best evidence that the fastsame phenomenon obtained with successive presentation is not attributable to response bias comes from the fact that there was no greater tendency to make false-"same" errors with successive presentation than with simultaneous presentation. In fact, the error patterns were very similar for the two methods of presentation. Thus, the reaction time advantage for same pairs with successive presentation, relative to simultaneous presentation, apparently is not due to an increased bias to respond "same." 


\section{POSITION EFFECTS FOR DIFFERENT PAIRS}

The different pairs in Experiments 1-3 differed at only one position. Responses to these pairs were classified according to the position of difference and reanalyzed. The mean error rates and reaction times are shown in Tables 2 and 3.

With simultaneous presentation, reaction time increased as the position of difference shifted from left to right. This increase was significant for fourletter strings in both Experiment 1 and Experiment 3 $[F(3,45)=30.6, p<.001$, and $F(3,30)=5.02, p<$ .025 , respectively], but not for either the two- or three-letter strings in Experiment 3. The lack of significance for the latter two string lengths in Experiment 3 is likely due to the small number of responses, inasmuch as Krueger (1984) has reported significant left-to-right increases for a similar experiment in which more responses were obtained. There were no reliable effects of position on the proportion of errors for simultaneous presentation in either Experiment 1 or Experiment 3 (Fs $<1.0$ ).

For successive presentation, the two-letter strings in Experiment 3 showed a slight, nonsignificant, advantage for the first position on both reaction time and error rate. Three-letter strings in Experiment 2 showed a significant left-to-right increase in reaction time $[F(2,30)=13.6, p<.001]$, with a trend toward fewer errors being made when the mismatching letter pair was in the left or center position than when it was in the right position $[F(2,40)=2.53, p<.10]$.

For three-letter strings in Experiment 3, as well as for four-letter strings in Experiments 1 and 3, there was no left-to-right increase in reaction time. Rather, responses were faster when the position of difference was the leftmost one than when it was any of the other positions, which were approximately equal. For the three-letter strings this tendency was nonsig-

Table 2

Mean Reaction Time (RT, in Milliseconds) and Proportion of Errors (PE) for "Different" Pairs in Experiments 1 and 2 as a Function of Position of Difference and Proportion Block

\begin{tabular}{|c|c|c|c|c|c|c|c|c|c|c|}
\hline \multirow[b]{3}{*}{ Position } & \multicolumn{10}{|c|}{ Proportion Block } \\
\hline & \multicolumn{2}{|c|}{$80 / 20$} & \multicolumn{2}{|c|}{$60 / 40$} & \multicolumn{2}{|c|}{$40 / 60$} & \multicolumn{2}{|c|}{$20 / 80$} & \multicolumn{2}{|c|}{$\mathbf{M}$} \\
\hline & RT & PE & RT & PE & RT & PE & RT & PE & RT & PE \\
\hline \multicolumn{11}{|c|}{ Experiment 1: Simultaneous Presentation } \\
\hline $\begin{array}{l}1 \\
2 \\
3 \\
4\end{array}$ & $\begin{array}{r}944 \\
1036 \\
1073 \\
1175\end{array}$ & $\begin{array}{l}.191 \\
.114 \\
.139 \\
.181\end{array}$ & $\begin{array}{r}989 \\
994 \\
1104 \\
1197\end{array}$ & $\begin{array}{l}.119 \\
.134 \\
.088 \\
.131\end{array}$ & $\begin{array}{r}986 \\
1034 \\
1089 \\
1213\end{array}$ & $\begin{array}{l}.118 \\
.117 \\
.093 \\
.113\end{array}$ & $\begin{array}{r}943 \\
982 \\
1111 \\
1201\end{array}$ & $\begin{array}{l}.067 \\
.053 \\
.061 \\
.072\end{array}$ & $\begin{array}{r}966 \\
1012 \\
1094 \\
1197\end{array}$ & $\begin{array}{l}.124 \\
.105 \\
.095 \\
.124\end{array}$ \\
\hline \multicolumn{11}{|c|}{ Experiment 1: Successive Presentation } \\
\hline $\begin{array}{l}1 \\
2 \\
3 \\
4\end{array}$ & $\begin{array}{l}622 \\
711 \\
722 \\
661\end{array}$ & $\begin{array}{l}.150 \\
.248 \\
.186 \\
.231\end{array}$ & $\begin{array}{l}640 \\
718 \\
701 \\
725\end{array}$ & $\begin{array}{l}.159 \\
.231 \\
.166 \\
.200\end{array}$ & $\begin{array}{l}663 \\
749 \\
749 \\
756\end{array}$ & $\begin{array}{l}.131 \\
.255 \\
.178 \\
.168\end{array}$ & $\begin{array}{l}633 \\
715 \\
711 \\
709\end{array}$ & $\begin{array}{l}.089 \\
.154 \\
.125 \\
.125\end{array}$ & $\begin{array}{l}640 \\
723 \\
721 \\
713\end{array}$ & $\begin{array}{l}.132 \\
.222 \\
.163 \\
.181\end{array}$ \\
\hline \multicolumn{11}{|c|}{ Experiment 2: Successive Presentation } \\
\hline $\begin{array}{l}1 \\
2 \\
3\end{array}$ & $\begin{array}{l}618 \\
689 \\
703\end{array}$ & $\begin{array}{l}.050 \\
.070 \\
.088\end{array}$ & $\begin{array}{l}688 \\
693 \\
726\end{array}$ & $\begin{array}{l}.072 \\
.058 \\
.120\end{array}$ & $\begin{array}{l}701 \\
737 \\
766\end{array}$ & $\begin{array}{l}.044 \\
.045 \\
.063\end{array}$ & $\begin{array}{l}625 \\
674 \\
711\end{array}$ & $\begin{array}{l}.035 \\
.029 \\
.039\end{array}$ & $\begin{array}{l}658 \\
698 \\
727\end{array}$ & $\begin{array}{l}.050 \\
.051 \\
.078\end{array}$ \\
\hline
\end{tabular}

Table 3

Mean Reaction Time (RT, in Milliseconds) and Proportion of Errors (PE) for "Different" Pairs in Experiment 3 as a Function of Position of Difference and String Length

\begin{tabular}{|c|c|c|c|c|c|c|c|c|c|}
\hline \multirow[b]{3}{*}{ Position } & \multicolumn{9}{|c|}{ String Length } \\
\hline & \multicolumn{2}{|c|}{1} & \multicolumn{2}{|c|}{2} & \multicolumn{2}{|c|}{3} & & \multicolumn{2}{|c|}{4} \\
\hline & RT & PE & RT & PE & RT & PE & & RT & PE \\
\hline \multicolumn{10}{|c|}{ Simultaneous Presentation } \\
\hline $\begin{array}{l}1 \\
2 \\
3 \\
4\end{array}$ & 541 & .014 & $\begin{array}{l}622 \\
628\end{array}$ & $\begin{array}{l}.031 \\
.014\end{array}$ & $\begin{array}{l}735 \\
770 \\
762\end{array}$ & $\begin{array}{l}.029 \\
.049 \\
.029\end{array}$ & : & $\begin{array}{r}883 \\
884 \\
974 \\
1022\end{array}$ & $\begin{array}{l}.046 \\
.057 \\
.063 \\
.068\end{array}$ \\
\hline \multicolumn{10}{|c|}{ Successive Presentation } \\
\hline $\begin{array}{l}1 \\
2 \\
3 \\
4\end{array}$ & 498 & .020 & $\begin{array}{l}547 \\
555\end{array}$ & $\begin{array}{l}.017 \\
.022\end{array}$ & $\begin{array}{l}583 \\
615 \\
613\end{array}$ & $\begin{array}{l}.008 \\
.040 \\
.028\end{array}$ & & $\begin{array}{l}685 \\
734 \\
708 \\
704\end{array}$ & $\begin{array}{l}.071 \\
.085 \\
.048 \\
.068\end{array}$ \\
\hline
\end{tabular}


nificant $[F(2,20)=2.36, p>.05]$, but it was paired with a corresponding nonsignificant tendency toward fewer errors for the left position $[F(2,20)=2.13, p>$ $.05]$. For the four-letter strings, this latency advantage for the leftmost position was significant in Experiment $1[F(3,45)=12.8, p<.001]$, but not in Experiment $3[F(3,30)=1.04, p>.05]$. Position also had a significant effect on errors in Experiment 1, with the pattern being complementary to that for reaction time $[F(3,45)=3.41, p<.025]$. That is, fewer errors were made when the difference was in the leftmost position than when it was in any of the other three.

To summarize, with simultaneous presentation, there is a consistent left-to-right position effect. With successive presentation, the position effect is primarily one of an advantage for the first position relative to the others, with this pattern being particularly clear for four-letter strings.

One additional outcome of interest regarding the position effects is that in both Experiment 1 and Experiment 2 there was no significant interaction of position with proportion block for either error rate or reaction time (Fs $<1.40$ ). That is, the position effects remained relatively consistent as the bias to respond "same" (or "different") varied. If subjects were performing a consistent left-to-right serial comparison or if completion order was always consistent, position should have interacted with proportion block, particularly on the error-rate measure.

\section{GENERAL DISCUSSION}

The two methods used in the present study provide converging evidence regarding the role of response bias in the fast-same phenomenon obtained with multiletter pairs. For simultaneously presented letter strings, the fast-same phenomenon that is usually obtained (e.g., Eichelman, 1970; Proctor \& Hurst, 1982; Silverman, 1973) appears to be attributable entirely to response bias. With the bias manipulation used in Experiment 1, correct "different" responses were faster overall than were correct "same" responses, even though false-"same" errors outnumbered false-"different" errors. With the procedure designed to minimize false-"same" errors in Experiment 3, "different" responses again were faster than "same" responses, and the effect of string length was much less on the latency of the former responses than on that of the latter. These results, obtained for simultaneously presented strings, are generally consistent with analytic, self-terminating models of the comparison process. More importantly, regardless of the particular nature of the processing operations, there seems to be no need to invoke a separate process that is specific to same pairs, when the multiletter strings are presented simultaneously.
In contrast to the results obtained with simultaneous presentation, the fast-same phenomenon was still apparent in the present experiments when the letter strings within each pair were presented successively. Several results argue that the fast-same effect obtained with successive presentation is not due to response bias. First, the fast-same effect apparent for three-letter strings in Experiment 2 was over and above that attributable to the influence of the bias manipulation, even though there was no differential tendency to make incorrect "same" or "different" responses. Second, when string length was manipulated in Experiment 3, a large increment in false"same" errors occurred only for four-letter strings. However, the fast-same effect was equally evident for strings of lengths 1-3. Third, in both Experiment 1 and Experiment 3, the tendency to make false-"same" errors rather than false-"different" errors was no greater for successive than for simultaneous presentation, yet the fast-same phenomenon was apparent for the former method of presentation but not for the latter.

Interestingly, results consistent with our findings have been obtained in two studies that used a decision rule that is the reverse of the more standard one used in the present experiments (Silverman \& Goldberg, 1975; Taylor, 1976). With the reversed rule, subjects respond "same" if the strings match at one or more positions and "different" only if they differ at all positions. This rule requires that the "same" response be made to pairs that differ at up to $\mathrm{N}-1$ positions, as well as to identical pairs. Any bias to terminate prematurely is switched primarily to the "different" response because this response is appropriate only if mismatches occur at all positions. Silverman and Goldberg found no reaction time advantage for identical pairs in the reversed task using simultaneous presentation, whereas Taylor did find an advantage using successive presentation. Thus, these results also suggest that the fast-same effect customarily obtained with simultaneous presentation is attributable to response bias, whereas that obtained with successive presentation is not.

Proctor (1981) proposed that for successively presented stimuli, the fast-same phenomenon is due in part to facilitation in the encoding of the second stimulus when it is physically identical to the first. Evidence for this facilitation principle was obtained by demonstrating that the commonly obtained reactiontime advantage for responding "same" to physically identical pairs (e.g., DD), relative to responding "same" to name-identical pairs (e.g., Dd), was also evident when the second letter had only to be named. The second of two physically identical letters also was named faster than a letter preceded by a neutral cue, indicating that the primary influence of a preceding letter is to benefit the encoding of the sub- 
sequent letter when the two are physically identical. This facilitation for physically identical pairs was not apparent in the naming task when the pair of letters was presented simultaneously. Proctor noted that both studies of single-letter matching and studies of multiletter matching show an enhanced fast-same effect for successively presented stimuli, as predicted by the facilitation principle.

Subsequent studies have provided even stronger support for the facilitation principle. Krueger (1983) emphasized that, in addition to predicting that the fast-same phenomenon should be greater with successive presentation than with simultaneous presentation, the facilitation principle also predicts that the benefit will occur without the expense of a relative increase in false-"same" errors. This prediction has been supported in studies of single-letter matching (Chignell \& Krueger, 1984; Krueger, 1983; Proctor \& Rao, 1983), as well as in the multiletter-matching experiments described in the present article. Moreover, tests of several alternative accounts of the enhanced fast-same effect that is obtained with successive presentation have proved negative (Chignell \& Krueger, 1984; Krueger, 1983). Thus, current evidence strongly suggests that facilitation in the encoding of the second of two physically identical stimuli is a primary contributor to the fast-same phenomenon obtained with successive presentation.

For multiletter strings, the facilitation in encoding could have either an analytic or a bolistic basis (i.e., it could occur either at the level of matching component letters or at the level of the entire string). Proctor (1981) and Proctor and Hurst (1982) have advocated the analytic viewpoint that processes operating at the level of the component letter pairs are the primary determinants of the results obtained with multiletter strings. This viewpoint is most strongly supported by the finding that the difference in reaction time to physically identical and nameidentical single-letter pairs is similar to the difference in slopes obtained for multiletter pairs of these two types when reaction time is plotted as a function of string length (Proctor \& Hurst, 1982).

Taylor (1976), on the other hand, has suggested that the facilitation obtained with multiletter strings may reflect a holistic benefit that occurs only when no mismatching pairs are present. His finding that identical strings show facilitation relative to nonidentical strings in the reversed task (respond "same" if one or more positions match) is more consistent with the holistic viewpoint. That is, because the "same" response can be based on a single matching letter pair in the reversed task, little advantage (other than that due to earlier termination of the comparison process) would be expected for identical strings unless there was a benefit resulting from all letter pairs being the same. Thus, at present, the evidence does not clearly indicate either an analytic or a holistic basis for the facilitation that occurs with successively presented. multiletter strings.

Although we have argued that there is a fast-same phenomenon with successive presentation that cannot be attributed to subjects prematurely responding "same" before a mismatch is detected, there is evidence in Experiments 1 and 2 suggesting that such premature terminations do occur for both simultaneous and successive presentation conditions. Response bias was manipulated in those experiments by varying the proportion of trials on which same pairs occurred. According to nonexhaustive, analytical models of the comparison, the increase in false"same" errors that occurs as bias shifts progressively toward the "same" response should reflect an increasing tendency to terminate the comparison prematurely. This increasing tendency to terminate prematurely would affect correct reaction times for both "same" and "different" responses, but the former would be affected more than the latter.

The basis for this prediction can be seen most readily from an example using the standard serial self-terminating model. If subjects were to adopt a criterion of responding "same" to four-letter strings when a mismatch has not been located in the first three comparisons, rather than waiting to complete the fourth comparison, correct "same" reaction times would be reduced by the amount of time necessary to perform this last comparison. Correct "different" reaction times would also be decreased, on the average, because any strings for which the mismatching letter pair was not among the first three comparisons would be incorrectly judged as "same." However, because each additional comparison adds only half of a comparison to the mean "different" reaction time (because of the self-terminating feature), the resulting decrease would be only half of that for "same" reaction times. Consistent with the premature termination hypothesis, the proportion block manipulation in Experiments 1 and 2 had a much greater influence on "same" than on "different" reaction times.

A counterintuitive prediction also apparent from the example is that correct "different" reaction times should decrease as the bias to respond "same" increases. Examination of Figures 1 and 2 indicates that this predicted result was obtained across the $40 / 60,60 / 40$, and $80 / 20$ blocks in both Experiments 1 and 2. Moreover, the relative decreases for "different" and "same" reaction times across these blocks were consistent with expectations. "Different" reaction times decreased less than "same" reaction times in all cases as the bias shifted toward "same," with the ratios of the slopes of the bestfitting lines to "different" and "same" reaction times being .24 for simultaneous presentation in Ex- 
periment 1 and .47 and .57 for successive presentation in Experiments 1 and 2, respectively.

The failure of "different" reaction times for the 20/80 block, in which different pairs predominated, to conform to the pattern evident for the other three blocks is not problematical for the premature termination account. When the probability of a different pair is high, subjects likely adopt a strategy of responding "different" on at least some prematurely terminated trials. More problematical for the premature termination hypothesis is that no false-"different" errors are predicted, other than possibly when different pairs predominate greatly, because "same" is the default response. The relatively high proportion of false-"different" errors made in Experiments 1 and 2 indicates, therefore, that there is a source of errors other than premature termination. Thus, the results of our manipulation of bias are consistent with the conclusion of Taylor (1976) that many errors in multiletter matching occur due to subjects' prematurely responding "same," although there are other sources. Moreover, our results are also in agreement with Taylor's conclusion that although such premature terminations occur, they cannot account for the fast-same phenomenon obtained with successive presentation.

Ratcliff and Hacker $(1981,1982)$ warned that response bias could confound the relationship between "same" and "different" reaction times. The present study indicates that their warning is well founded. There appears to be no fast-same phenomenon for simultaneously presented multiletter strings when the contribution of response bias is minimized. However, Ratcliff and Hacker's general conclusion that the fast-same phenomenon reflects "nothing more" than response bias is not justified. With successive presentation, there is a reliable fast-same phenomenon for both single-letter (Proctor \& Rao, 1983) and multiletter pairs that cannot be explained without recourse to some mechanism other than the "same". "different" response criteria.

In his most recent review of the fast-same phenomenon (which focused primarily on studies using successive presentation), Nickerson (1978) concluded:

Response biases, when they occur, can undoubtedly affect the relative speeds of same and different responses .... . However, the evidence seems to suggest that there is a residual difference between same and different RT to be explained after such "peripheral" factors have been taken into account (p. 85).

We agree with Nickerson's conclusion and believe that facilitation in the encoding of the second of two successively presented, identical stimuli accounts for much of this residual difference.

\section{REFERENCES}

BAmber, D. (1969). Reaction times and error rates for "same""different" judgments of multidimensional stimuli. Perception \& Psychophysics, 6, 169-174.

Bamber, D., \& PAine, S. (1973). Information retrieval processes in "same"-" different" judgments of letter strings. In S. Kornblum (Ed.), Attention and performance IV (pp. 477-496). New York: Academic Press.

Chigneld, M. H., \& Krueaer, L. E. (1984). Further evidence for priming in perceptual matching: Temporal, not spatial, separation enhances the fast-same effect. Unpublished manuscript.

Coltheart, M., \& CuRthoYs, I. (1968). Short-term recognition memory for pitch: Effect of a priori probability on response times and error rates. Perception \& Psychophysics, 4, 85-89.

Downing, B. D. (1971). Response probabilities and "samedifferent" reaction times. Perception \& Psychophysics, 9, 213-215.

Eichelman, W. H. (1970). Familiarity effects in the simultaneous matching task. Journal of Experimental Psychology, 86, 275-282.

Eriksen, C. W., O'Hara, W. P., \& Eriksen, B. A. (1982). Response competiton effects in same-different judgments. Perception \& Psychophysics, 32, 261-270.

GrILL, D. P. (1971). Variables influencing the mode of processing of complex stimuli. Perception \& Psychophysics, 10, 51-57.

Henderson, L., \& Henderson, S. E. (1975). Visual comparison of words and random letter strings: Effects of number and position of letters different. Memory \& Cognition, 3, 97-101.

KRUEGER, L. E. (1977). A theory of perceptual matching (Tech. Rep. No. 1). Columbus: Ohio State University, Human Performance Center.

Krueger, L. E. (1978). A theory of perceptual matching. Psychological Review, 85, 278-304.

Krugger, L. E. (1983). Probing Proctor's priming principle: The effect of simultaneous and sequential presentation on samedifferent judgments. Journal of Experimental Psychology: Learning, Memory, and Cognition, 9, 511-523.

KRUeGer, L. E. (in press). Self-termination in same-different judgments: Multiletter comparison with simultaneous and sequential presentation. Journal of Experimental Psychology: Learning, Memory, and Cognition, 10, 271-284.

KRUEGer, L. E., \& Shapiro, R. G. (1981a). Intertrial effects of same-different judgements. Quarterly Journal of Experimental Psychology, 33A, 241-265.

KRUEGer, L. E., \& Shapiro, R. G. (1981b). A reformulation of Proctor's unified theory for matching-task phenomena. Psychological Review, 88, 573-581.

Nickerson, R. S. (1965). Responses for "same"-"different" judgments. Perceptual and Motor Skills, 20, 15-18.

Nickerson, R. S. (1972). Binary-classification reaction time: A review of some studies of human information-processing capabilities. Psychonomic Monograph Supplements, 4(17, Whole No. 65).

Nickerson, R. S. (1978). On the time it takes to tell things apart. In J. Requin (Ed.), Attention and performance VII (pp. 77-88). Hillsdale, NJ: Erlbaum.

Procton, R. W. (1981). A unified theory for matching-task phenomena. Psychological Review, 88, 291-326.

Procton, R. W., \& HuRst, P. W. (1982). A parametric investigation of multiletter matches. Perception \& Psychophysics, 32, 75-84.

Proctor, R. W., Rao, K. V. (1982). On the "misguided" use of reaction-time differences: $A$ discussion of Ratcliff and Hacker (1981). Perception \& Psychophysics, 31, 601-602.

Proctor, R. W., RAo, K. V. (1983). Evidence that the samedifferent disparity in letter matching is not attributable to response bias. Perception \& Psychophysics, 34, 72-76.

RATcliff, R. (1981). A theory of order relations in perceptual matching. Psychological Review, 88, 552-572. 
RATCLIFF, R., \& HACkER, M. J. (1981). Speed and accuracy of same and different responses in perceptual matching. Perception \& Psychophysics, 30, 303-307.

Ratclift, R., \& Hacker, M. J. (1982). On the misguided use of reaction-time differences: A reply to Proctor and Rao (1982). Perception \& Psychophysics, 31, 603-604.

Silverman, W. P. (1973). The perception of identity in simultaneously presented complex visual displays. Memory \& Cognition, 1, 459.466.

Silverman, W., \& Goldbero, S. (1975). Further confirmation of same vs. different processing differences. Perception \& Psychophysics, 17, 189-193.
TAYLOR, D. A. (1976). Effect of identity in the multiletter matching task. Journal of Experimental Psychology: Human Perception and Performance, 2, 417-428.

\section{NOTE}

1. Eichelman (1970) reported error rates collapsed across type of pair, stating that only the string length effect was significant. However, the predominance of false-"same" errors at string lengths of four or greater is so pervasive in other studies that we consider it likely that the $9.1 \%$ error rate for four-letter strings in his study reflects primarily false-"same" errors.

(Manuscript received December 21, 1983;

revision accepted for publication March 19, 1984.) 